\title{
On Cremona transformations of prime order
}

\section{Sur les transformations de Cremona d'ordre premier}

\author{
Arnaud Beauville \\ Laboratoire J.-A. Dieudonné, UMR 6621 du CNRS, Université de Nice, Parc Valrose, F-06108 Nice cedex 2 \\ Jérémy Blanc \\ Section de Mathématiques, Université de Genève, 2-4 rue du Lièvre, Case postale 240, CH-1211 Genève 24
}

\begin{abstract}
We prove that an automorphism of order 5 of the Del Pezzo surface $S_{5}$ of degree 5 is conjugate through a birational map $S \rightarrow \mathbb{P}^{2}$ to a linear automorphism of $\mathbb{P}^{2}$. This completes the classification of conjugacy classes of elements of prime order in the Cremona group.

\section{Résumé}

Nous démontrons qu'un automorphisme d'ordre 5 de la surface de Del Pezzo $S$ de degré 5 est conjugué via une application birationnelle $S \rightarrow \mathbb{P}^{2}$ à un automorphisme linéaire de $\mathbb{P}^{2}$. Cette observation complète la classification des classes de conjugaison d'élements d'ordre premier dans le groupe de Cremona.
\end{abstract}

\section{The result}

The Cremona group $\operatorname{Bir}\left(\mathbb{P}^{2}\right)$ is the group of birational transformations of $\mathbb{P}_{\mathbb{C}}^{2}$, or equivalently the group of $\mathbb{C}$-automorphisms of the field $\mathbb{C}(x, y)$. There is an extensive classical literature about this group, in particular about its finite subgroups; we refer to the introduction of $[\mathrm{dF}]$ for a list of references.

The classification of conjugacy classes of elements of prime order $p$ in $\operatorname{Bir}\left(\mathbb{P}^{2}\right)$ has been given a modern treatment in [B-B] for $p=2$ and in $[\mathrm{dF}]$ for $p \geq 3$. Let us recall the main results. The linear transformations of given order are contained in a unique conjugacy class (see the Proposition below). Apart from these there are three families of conjugacy classes of involutions (the famous de Jonquières, Bertini and Geiser involutions), then two families of conjugacy classes of order 3, given by automorphisms of special Del

Email addresses: beauville@math.unice.fr (Arnaud Beauville), Jeremy.Blanc@math.unige.ch (Jérémy Blanc). 
Pezzo surfaces of degree 3 and 1 respectively ${ }^{1}$. Every transformation of prime order $\geq 7$ is conjugate to a linear automorphism. As for conjugacy classes of order 5, there is at least one family, given by automorphisms of a special Del Pezzo surface of degree 1 (example E3 in [dF]).

The following result completes the classification:

Theorem.- Every conjugacy class of order 5 in $\operatorname{Bir}\left(\mathbb{P}^{2}\right)$ is either of the above type, or is the class containing the linear automorphisms.

Most of the work has been done already in $[\mathrm{dF}]$, where it is proved that there can be only one more conjugacy class of order 5, namely that of an automorphism $\sigma$ (of order 5) of the Del Pezzo surface $S_{5} \subset \mathbb{P}^{5}$. We will prove in the next section that $\sigma$ is conjugate to a linear automorphism.

The theorem, together with the classification in $[\mathrm{dF}]$, has the following consequence:

Corollary.- A birational transformation of prime order is not conjugate to a linear automorphism if and only if it fixes some non-rational curve.

Observe that this does not hold for transformations of composite order: the automorphism $\sigma$ of the cubic surface $x^{3}+y^{3}+z^{3}+t^{3}=0$ in $\mathbb{P}^{3}$ given by

$$
\sigma(x, y, z, t)=(y, x, z, \rho t) \quad \text { with } \rho^{3}=1, \rho \neq 1
$$

has only 4 fixed points, while $\sigma^{2}$ fixes the elliptic curve $t=0$. Thus the conjugacy class of $\sigma$ in $\operatorname{Bir}\left(\mathbb{P}^{2}\right)$ cannot contain a linear automorphism.

\section{The proof}

Let us first recall why linear automorphisms of the same (finite) order are conjugate:

Proposition.- Let $n$ be a positive integer. Two linear automorphisms of order $n$ are conjugate in $\operatorname{Bir}\left(\mathbb{P}^{2}\right)$.

Proof : Let $T=\mathbb{C}^{*} \times \mathbb{C}^{*}$ be the standard maximal torus of $P G L_{3}$. We can view $T$ as a Zariski open subset of $\mathbb{P}^{2}$, so its automorphism group $G L(2, \mathbb{Z})$ embeds naturally in $\operatorname{Bir}\left(\mathbb{P}^{2}\right)$ : an element $\left(\begin{array}{ll}a & b \\ c & d\end{array}\right)$ of $G L(2, \mathbb{Z})$ corresponds to the Cremona transformation $(x, y) \mapsto\left(x^{a} y^{b}, x^{c} y^{d}\right)$. The image of $G L(2, \mathbb{Z})$ in $\operatorname{Bir}\left(\mathbb{P}^{2}\right)$ normalizes $T$, and its action on $T$ by conjugation is just the original action of $G L(2, \mathbb{Z})$ on $T$.

We want to prove that two elements of order $n$ of $T$ are conjugate under this action. The kernel of the multiplication by $n$ in $T$ is $(\mathbb{Z} / n)^{2}$; any element of order $n$ in the $\mathbb{Z} / n$-module $(\mathbb{Z} / n)^{2}$ is part of a basis, which implies that it is conjugate to $(1,0)$ under the action of $S L(2, \mathbb{Z} / n)$. Since the natural map $S L(2, \mathbb{Z}) \rightarrow S L(2, \mathbb{Z} / n)$ is surjective, this proves our result.

Proof of the theorem:

Let $V$ be a 6 -dimensional vector space, and $S \subset \mathbb{P}(V)$ a Del Pezzo surface of degree 5 . It admits an automorphism $\sigma$ of order 5 , unique up to conjugation, which comes from a linear automorphism $s$ of $V$.

The automorphism $\sigma$ has at least one fixed point $p$ (otherwise we would have a smooth étale covering $S \rightarrow S /\langle\sigma\rangle$ of degree 5 , so that $1=\chi\left(\mathcal{O}_{S}\right)=5 \chi\left(\mathcal{O}_{S /\langle\sigma\rangle}\right)$, a contradiction). The tangent plane $\mathbb{P}\left(T_{p}\right)$ to $S$ at $p$ corresponds to a 3 -dimensional vector subspace $T_{p} \subset V$. Since $s$ preserves $T_{p}$, it induces a linear automorphism $\bar{\sigma}$ of $\mathbb{P}^{2}=\mathbb{P}\left(V / T_{p}\right)$; we have a commutative diagram

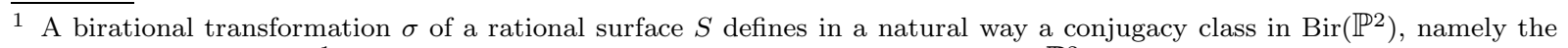
set of elements $\pi \circ \sigma \circ \pi^{-1}$ where $\pi$ runs through the set of birational maps $S \rightarrow-\rightarrow \mathbb{P}^{2}$.
} 


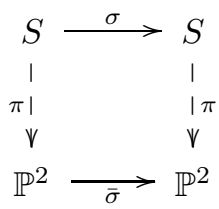

where $\pi: S \rightarrow \mathbb{P}^{2}$ is the projection from $\mathbb{P}\left(T_{p}\right)$.

We claim that $\pi$ is birational, so that it conjugates $\sigma$ to the linear automorphism $\bar{\sigma}$ of $\mathbb{P}^{2}$. Identify $S$ with $\mathbb{P}^{2}$ blown up at 4 points $p_{1}, \ldots, p_{4}$ in general position. The map $\pi: S \rightarrow \mathbb{P}^{2}$ is given by the linear system $|\mathcal{C}|$ of cubics passing through $p_{1}, \ldots, p_{4}$ and singular at $p$. Note that the 5 points $p_{1}, \ldots, p_{4}, p$ are again in general position (that is, no 3 of them are collinear). Indeed this means that $p$ does not belong to any line in $S$. But $S$ contains 10 lines, on which $\langle\sigma\rangle$ acts with 2 orbits; if $p$ lies on a line it must lie on 5 of them, which is impossible.

Thus $\pi$ is the de Jonquières transformation of order 3, a classical instance of birational transformation of $\mathbb{P}^{2}$ (see e.g. [S-R], VII, 7.2). This achieves the proof of the theorem.

Remark.- We can make the result completely explicit. We use the coordinates system on $\mathbb{P}^{2}$ such that $\left(p_{1}, \ldots, p_{4}\right)$ is a projective frame; as in $[\mathrm{dF}]$, we can view $\sigma$ as the lift over $S$ of the birational transformation $(x: y: z) \mapsto(x(z-y): z(x-y): x z)$ of $\mathbb{P}^{2}$. This transformation has two fixed points $\left(\omega: 1: \omega^{2}\right)$, with $\omega=\frac{1}{2}(1 \pm \sqrt{5})$. Choosing one of these fixed points we find that $\sigma$ is conjugate by the involutive transformation

$$
(x: y: z) \mapsto\left((x-\omega y)(y-z)(z-\omega x):\left(\omega^{-1} x-y\right)\left(\omega^{2} y-z\right)(z-x):(x-y)\left(\omega^{2} y-z\right)(z-\omega x)\right)
$$

to the linear automorphism of $\mathbb{P}^{2}$ given by the matrix

$$
\left(\begin{array}{ccc}
0 & 1 & -\omega^{-2} \\
-\omega^{-1} & 1 & 0 \\
0 & 1 & 0
\end{array}\right) .
$$

\section{References}

[B-B] L. Bayle, A. Beauville: Birational involutions of $\mathbb{P}^{2}$. Kodaira's issue, Asian J. Math. 4 (2000), 11-17.

[dF] T. de Fernex: On planar Cremona maps of prime order. Nagoya Math. J. 174 (2004). Preprint math.AG/0302175

[S-R] J. Semple, L. Roth: Introduction to Algebraic Geometry. Oxford, at the Clarendon Press, 1949. 